\title{
Detlef Lehnert Wie desintegrativ war die Weimarer Reichsverfassung?
}

Der Autor will veraltete Fragestellungen zur Weimarer Republik mit neu akzentuierten Anworten versehen. Besonders beschäftigt er sich mit der verbreiteten These, die Weimarer Verfassung habe zu wenig zu integrieren vermocht. Der Autor zeigt, daß sich dabinter nicht selten politisch die Tatsache verbarg, daß bestimmte politische Parteien die neue Verfassungsordnung ablehnten. Mit der These von der mangelnden Integrationskraft der Verfassung konnten jene Staatsrechtslehrer, die vor 1933 nicht als Verfechter der politischen Demokratie galten, ibre nach 1945 fragwürdig erscheinende Grundhaltung rechtfertigen.

Die Red.

So lauter gewissermaßen antithetisch zum Tagungstitel ${ }^{x}$ die vorgegebene Fragestellung; derart vieldeutig ist sie nur deshalb belassen worden, um die folgenden Problemebenen auffächern zu können: Einem möglichen desintegrativen Potential soll erstens - im politischen Willensbildungsprozeß des Zustandekommens dieser Verfassung nachgespürt werden. Zweitens lassen sich die beschlossenen Textformulierungen befragen, inwiefern deren Normenstruktur von unbewältigten Widersprüchen geprägt wurde. Eine dritte Perspektive wird in den Blick zu nehmen haben, ob zentrifugale Tendenzen erst aus einer Verfassungspraxis resultierten, die frühzeitig Ausgangskompromisse sprengte und sogar normdurchbrechend agierte. Viertens darf nicht ungeprüft bleiben, ob gerade auch von Hauptströmungen der Weimarer Staatsrechtslehre jene desintegrativen Elemente in die Verfassungsdeutung hineinprojiziert worden sind. Ein fünfter Aspekt hat gegen die Beweiskraft einer desolaten Verfassungslage der frühen zoer Jahre auch die Gegenperspektive zu erwägen: ob solche Zustände, statt als Beleg für desintegrative Elemente der Verfassung zu dienen, nicht eher die Konsequenz nahelegen, die Zerstörung einer Weimarer Republik bereits vor $\mathrm{dem}$ Jahre 1933 zu datieren. Und sechstens gilt es zu überdenken, inwiefern das »Bonn ist nicht Weimar «-Syndrom manche retrospektive Denkschablone zu fixieren vermochte.

\section{I.}

Beginnen wir also mit dem Willensbildungsprozeß, der im Weimarer Verfassungswerk kodifiziert wurde. Die im Januar 1919 erstmals von allen Frauen und Männern ab 20 Jahren gewählte Verfassunggebende Nationalversammlung verkörperte geradewegs den Idealtypus eines demokratischen Kreationsorgans: Nicht auf den

\footnotetext{
I Dieser Text entspricht weitgehend dem Wortaut eines Vortrags am 23. 10. 1998 auf der Tagung \# Integration durch Verfassung * in Dresden, jedoch erganzt um die Fußnoten mit Zitatbelegen, Literaturhinweisen und einzelnen vertiefenden Gesichtspunkten.
} 
»ewigen Bund» von Landesfürsten, wie dies einleitend zur Reichsverfassung von I $87 \mathrm{r}$ hieß, sondern auf das politische Selbstorganisationsrecht der erwachsenen Bevölkerung waren nunmehr die Repräsentativkörperschaften gegründet. Der herrschaftlich-obrigkeitsstaatliche Integrationstypus des Kaiserreiches konnte sich in Friedenszeiten mit passiver Massenloyalität begnügen. Den bürgergenossenschaftlich-volksstaatlichen Integrationstypus der Weimarer Republik, wie ihn der Verfassungsautor Hugo Preuß präsentierte ${ }^{2}$, mußte jedoch eine aktive Trägerschaft konstituieren. Gerade wegen dieser notwendigen massengesellschaftlichen Verankerung war die Weimarer Koalition von SPD, DDP und Zentrum in sich heterogener strukturiert als das nationalliberal-freikonservative Honoratiorenkartell der Bismarckära. Nach zeitgenössischer Diagnose des Wiener Verfassungsautors Hans Kelsen stellte ein Volk in freigesetzter Pluralität mehr "ein Bündel von Gruppen, als eine zusammenhängende Masse eines und desselben Aggregatzustandes« dar; aus solchem demokratischen Blickwinkel war dies aber keineswegs ein desintegrativer Störfaktor: "Gerade darin liegt ja ein Vorzug der Demokratie und ihres Majoritätsprinzipes», so argumentiert Kelsen', »daß sie bei größtmöglicher Einfachheit der Organisation doch immerhin eine gewisse politische Integration der Staatsgesellschaft gewährleistet. Die Praxis der Demokratie entwickelt eben schrittweise oder hat doch wenigstens die Tendenz, dies zu tun, was ihre Theorie schon voraussetzt« - nämlich prozedural integrationsformend zu wirken.

Ein weiteres Stichwort von Preuß aufgreifend, hatte es zunächst »Aufgabe des Verfassungsentwurfs « zu sein, »den politischen und staatsrechtlichen Niederschlag der Revolution festzulegen» ${ }^{4}$, also die normative Kraft des Systemwechsels als Rahmen künftiger Wahl-, Gesetzgebungs- und Regierungsentscheidungen zu kodifizieren. Vor Eintritt in die öffentliche Erörterung der Ende Januar I $9 \times 9$ veröffentlichten Textfassung sind allerdings weitere Festlegungen erfolgt, denen Franz L. Neumann rückblickend Sozialvertragscharakter beimessen wolltes: Das Stinnes-Legien-Abkommen regelte neben den Verbands- auch die Arbeitsbeziehungen; der Sicherheitspakt Ebert-Groener brachte mit dem Offizierskorps eine Herrschaftsgruppe des Kaiserreichs ins Machtgefüge zurück; die Kompetenzregelungen auf den ReichLänder-Konferenzen stoppten Neuordnungsziele wie eine Aufgliederung Preußens und stärkten die Position der überkommenen Bürokratien; hinzu trat in einer Anfangsphase der parlamentarischen Willensbildung der Mitbestimmungskompromiß in der Betriebsrätefrage nach den streikintensiven Märzunruhen. Dies alles vollzog sich freilich nicht im politik- und parteifreien Raum; ganz im Gegenteil waren die genannten Eckpfeiler ein integraler Bestandteil des neusortierten parteiendemokratischen Macht- und Richtungskampfes: Ein Arbeitgeberchef Stinnes und der Gewerkschaftsvorsitzende Legien blieben einflußreiche DVP-bzw. SPD-Politiker; die politische Laufbahn des ersten Reichspräsidenten Ebert ist bekannt, selbst General Groener wurde in späteren Jahren zum DVP-nahen Wehr- und Innenminister gouvernementalisiert; die eigensinnigen Länderdelegierten waren in unterschiedlichen

2 Im Sinne einer bislang vernachlassigten Darstellung und Interpretation scines Gesamtwerks kann verwiesen werden auf Detlef Lehnert, Verfassungsdemokratie als Burgergenossenschaft. Politisches Denken, Offencliches Recbe und Geschichtsdeutungen bei Hugo Preuß, Baden-Baden 1998.

3 Hans Kelsen, Vom Wesen und Wert der Demokratic, Tübingen r 220, S. 26, 28; die Berücksichrigung der Gedanken Kelsens grünklet sich hier, neben dem bahnbrechenden W/erk von Horst Dreicr, Rechtslehre, Staatssoziologic und Demokratictheorie bei Hans Kelsen, 2. Aufl. Baden-Baden I99o, zugleich auf eigene Sudien: Detlef Lehnert, wStaatslehre ohne Staat«? Zum kritischen Auftrag der rechts- und demokratietheoretischen Konzeption gegenuber deutschen Staatsvorstellungen, in: IfS-Nachrichsen 6/1998, S. 7-46.

4 Zit. nach Ernst Rudolf Huber (Hrsg.), Dokumente zur deutschen Verfassungsgeschichte, Bd. 3, Sturtgart I 966, S. 29.

5 Vgi. Franz L. Neumann, Die Herrschaft des Gesetzes. Eine Untersuchung zum Verhältnis von politischer Theorie und Rechrssystem in der Konkurrenzgese!lschaft, Frankfure a.M. 1980 (engl. 1936), S. 319 f. 
Konfigurationen die regionalen Vertreter auch jener Parteien, die auf Reichsebene stärker unitarisierende Programmziele verfolgten; das Betriebsräteproblem durfte im wesentlich als Geschwisterstreit zwischen SPD und USPD gelten. Der bürgergenossenschaftlich-volksstaatliche Integrationstypus war eben notwendig parteiendemokratisch ausgelegt. In den Verfassungstext wurde dies zwar nicht hineinformulicrt, aber mit Ausnahme deutschnationaler Staatsrechtler wie Heinrich Triepel ${ }^{6}$ fand sich für die These einer impliziten Parteienverankerung entgegen beharrlich anderslautenden Darstellungen sozusagen eine Große Deutungskoalition?: von Sozialdemokraten wie Gustav Radbruch bis zu Rechtsliberalen wie Gerhard Leibholz, mit ausdrücklicher Unterstützung auch durch Kelsen und Preuß $\beta^{8}$.

II.

Ein Blick auf das Normengefüge der Weimarer Verfassung wird immanente und kontextuelle Deutungsebenen zu unterscheiden haben: Als Sinneinheit mit relativ eindeutigen Intentionen läßt sich nicht einmal die Erstfassung aus den Kommissionsprotokollen des Verfassungsbeirats rekonstruieren: Den machtvollen Reichspräsidenten, um das für »Weimar « folgenreichste Verfassungssyndrom herauszugreifen, wollte tarsächlich Max Weber, und zwar ganz bewußt als Kontinuitätsfaktor und "Gegengewicht" zum Parlament", um eigenberechtigres Handeln der inneren Verwaltung und des Regierens nach außen gegenüber dem Verbands- und Parteizugriff abzuschirmen. Solche Absichten teilten andere prominente Stimmen zugunsten der Präsidentschaftsrepublik, die ebenfalls aus nationalliberalen Traditionen zur DDP gefunden hatten, wie z. B. der Historiker Friedrich Meinecke, während der spätere Weimarer "Kronjurist « Gerhard Anschütz sich diesbezüglich zunächst reserviert äußerte. Des letzteren erst kürzlich erschienene Autobiographie hat aber, mit Verweis auf die Gesinnungsnähe zu Meinecke, Weber und dem früheren National-Sozialen Friedrich Naumann, die Absicht enthüllt, "das Werk von Weimar nicht so sehr als einen Umsturz denn als Fortentwicklung, Fortbildung der Verfassung des Kaiserreiches " $\mathrm{zu}$ interpretieren. ${ }^{10}$

Eben diese staatsrechtsdogmatische Gefangenschaft in Denkhorizonten der Bismarck-Verfassung teilte Preuß, wie auch sein Wiener Gegenstück Kelsen, überhaupt nicht. Daß »Weimar» geradewegs der Kontrastentwurf zum inkriminierten »Scheinkonstitutionalismus« der Bismarckära werden sollte, wurde in der Verfassungsdenkschrift und begleitenden Studien unmißverständlich formuliert. Insofern traf Walter Jellinek als Chronist des Verfassungswandels aus eigener nationalliberaler Distanz einen Kern der Sache, wenn er argwöhnte, Preuß habe im Konsens mit dem Rat der

6 Heinrich Triepcl, Die Staatsverfassung und die politischen Parteien, Berlin 1928; dessen kaiserzeitlich geprägte Grundthese vom »extrakonstitutionellen* Status der Parteien teilte bezeichnenderweise auch scin wichtigster DNVP-Fachkollege: Rudolf Smend, Verfassung und Verfassungsrecht, München 1928, S. I 37. 7 Vgl. Christoph Gusy, Die Lehre vom Parteienstaat in der Weimarer Republik, Baden-Baden 1993.

$8 \mathrm{Im}$ posthum veroffentlichten Kommentartext von Preuß findet sich ein deutiicher Beleg gegen die Legende vom parteienfeindlichen Weimarer Verfassungsgesetzgeber und ein kritischer Unterton hinsiehtlich der versäumten Klarheit: $n$ Es herrscht also noch immer die Regel, daß Verfassungen und Gesetze die Voraussetzung ihrer Wirksamkeit, das Parteiwesen, mit keiner Silbe erwähnen. Um die Repràsentarion der offentlichen Meinung des ganzen Volkes rechtlich zu organisieren, setzt diese rechtliche Organisation dic Selbstorganisation des Volkes in politischen Parteien stillschweigend voraus «; Hugo Preuß, Reich und Lander, Berlin 1928, S. 269.

9 Gemäß dieser Quelle: Max Weber, Gesamtausgabe, Bd. 16., Tubingen 1988, 85 f., 89 war hingegen in Entwurf von Preuß dem Kanzleramt ndie Stellung eines enghschen Premier-Ministers $\propto$ zugedacht, dic nur mit begrenzten Rechten eines Staatsoberhaupts vereinhart werden konnte.

ro Gerhard Anschütz, Aus mcinem Leben. Hrsgg. und eingel. von W. Pauly, Frankfurt a.M. 1993, S. 287. 
Volksbeauftragten bewußt wenig vom Bismarckreich übernehmen wollen und stattdessen an Leitbildern aus England, Frankreich, den USA und der Schweiz entlang seinen Textentwurf komponiert, wo er nicht dem Paulskirchenerbe folgen konnte." Gleichwohl der nationalliberalen Deutungstradition den Vorzug zu geben, durfte im Rahmen der Lehr- und Meinungsfreiheit als zulässige Wortmeldung gelten. Die Fortschreibungsthese vom Kaiserreich zur Republik jedoch der Weimarer Verfassung als deren verborgenen Sinngehalt zu unterlegen, ignoriert allzu offensichtlich das Wahlvotum zur Nationalversammlung: Neben 45 I $/ 2 \%$ sozialdemokratischen Stimmen können in jedem Falle hinreichende DDP- und Zentrumsanteile als Neuordnungspotential betrachtet werden. Die klare Mehrheit von 21 I gegen 89 Abgeordnete zugunsten der schwarz-rot-goldenen Fahne der 1848 er Tradition anstelle des kaiserzeitlichen Schwarz-weiß-rot bezeugte ebenso auf der Symbolebene diesen Gründungswillen zur demokratischen Alternative. In der symbolkonservativen Minorität befand sich allerdings neben ehemaligen Nationalliberalen auch die NaumannGruppe der DDP. Mit ihrer teils protestantisch, teils spätwilhelminisch-nationalistisch eingefärbten Stimmungslage gegen den Anschluß zum Westen eignen sich insofern auch die häufig überschätzten Naumann-Gefolgsleute mangels parlamentarischen Eigengewichts nicht als Kronzeugen für Geburtsfehler Weimars. ${ }^{12}$

Vor solchem Hintergrund der Gründungskonstellation lassen sich Kerngehalte des Weimarer Verfassungsprojekts intentions- und normengerechter skizzieren. Das Nebeneinander von Reichspräsident, Parlamentarismus und Volksentscheiden ist häufig als wesentlicher Desintegrationsfaktor für damaliges Staatsorganisationsrecht beschrieben worden. Im nur selten herangezogenen Verfassungskommentar von Preuß war jedoch die beabsichtigte Prioritätensetzung deutlich formuliert: "Die deutsche Republik wird durch die Reichsverfassung als mittelbare, repräsentative, und zwar parlamentarische Demokratie organisiert«; dieses Hauptprinzip der Regierungsform sollte lediglich modifizicrt sein »durch die unmittelbar demokratischen Einrichtungen des Volksentscheids und Volksbegehrens, der Volksabstimmung, der Wahl und Absetzung des Reichspräsidenten durch das Volk «. ${ }^{13}$ Die Hürde für wirksame Volksentscheide war mit über der Hälfte aller Stimmberechtigten ohnehin dermaßen hoch gelegt, daß inzwischen die relative Bedeutungslosigkeit dieses Verfassungsinstituts für die Weimarer Republik kaum mehr bestritten wird. Ein hartnäkkiges Vorurteil sieht die Richtlinienkompetenz des Kanzlers erst als Errungenschaft des Grundgesetzes von 1949; in Wirklichkeit fand sie sich nahezu wortgleich bereits im Weimarer Verfassungsartikel §6: „Der Reichskanzler bestimmt die Richtlinien der Politik und trägt dafür gegenüber dem Reichstag die Verantwortung, « Die instrumentelle Leitung der Politik sollte demnach, analog dem britischen Premierministeramt, im parlamentarischen Regierungssystem konzentriert sein, während der Reichspräsident mehr als Integrationssymbol der Außenvertretung und der inneren Einheit gedacht war. In der operativen Politik wurde eine spezifische Rolle für das Präsidentenamt nur als Integrationsreserve vorgesehen, etwa für den Fall einer gewaltsamen Lahmlegung des Regierungshandelns - oder wenn sich aus cinem fragmentierten Parteiensystem nicht selbständig ein richtlinienbestimmendes Kabinett ergab.

Was für die Normenstruktur dieses "Plan of Government " herauszuarbeiten ist, gilt auch für die Weimarer »Declaration of Rights « als zweite Säule klassischer Verfas-

I $\mathrm{Vgl}$. Walter Jellinek, Revolution und Reichsverfassung, in: Jahrbuch des öffentlichen Rechtes der Gegenwart $9 / 1920$, S. 47.

I 2 Die Ambivalenz dieser mnational-sozialen « Traditionsprägung wird auch in abwägenden Darstellungen klar ersichrlich: Peter Theiner, Sozialer Liberalismus und deutsche Weltpolitik: Friedrich Naumann im Wilhelminischen Deutschland (1860-1919), Baden-Baden 1983.

13 Preuß, Reich (Fn. 8), S. 49. 
sungsprogrammatik. Nur wenige Monate nach dem Verfassungsbeschluß insistierte Preuß in einem Beitrag für das weltbeachtete »Berliner Tageblatt « (25. 12. 1919) auf folgender Neuakzentuierung: "Die deutsche Demokratie betont notwendigerweise und mit Recht das soziale Moment stärker als die westlichen Demokratien. " Dieses Richtmaß einer, zumal nach den Kriegsverwerfungen, unverzichtbaren sozialpolitischen Flankierung eines demokratischen Integrationsprozesses wurde von einer Nationalversammlungsmehrheit aus Sozialdemokraten, Sozialliberalen und Sozialkatholiken vorgegeben. Seinen institutionellen Ausdruck fand das Integrationskonzept einer Parität von Kapital und Arbeit nicht zuletzt im Räteartikel r6s WRV und der Erwähnung verschiedenster Sozialgruppen im Verfassungstext. In geistig-kultureller Hinsicht war die Weimarer Verfassung den Grundsätzen der Pluralität verpflichtet; dies konnte als Schrumpfform des Rückzugs auf den »dilatorischen Formelkompromiß ${ }^{14}$ nur brandmarken, wer gleich Carl Schmitt einem weltanschaulich illiberalen Ordnungsdenken anhing. In einer Nationalversammlung aus einer laizistischen Hälfte und je einem knappen protestantischen und katholischen Viertel hieß das Leitprinzip folgerichtig weltanschauliche Neutralität des Verfassungstaates, der zugleich diversen Teilkulturen ihre Autonomieräume und Mitwirkungschancen garantierte. ${ }^{15}$

III.

In den ersten Jahren entsprach die politische Realität der Normenprojektion tatsächlich mehr als gemeinhin angenommen. Ein der Apologetik ganz unverdächtiger Zeuge ist dafür Otto Kirchheimer, der rückblickend die »neue parlamentarischdemokratische Rechtsgemeinschaft « der Weimarer Republik auf bestimmte gesellschaftspolitische Integrationskonzepte angewiesen sah: »Das für eine solche Rechtsgemeinschaft notwendige Minimum an gemeinsamen Grundeinsichten war an die Existenz eines liberalen, zu einem selbständigen Ausgleich mit der Arbeiterschaft fähigen Bürgertums gebunden ", schrieb Kirchheimer unter dem Eindruck späterer Verfassungskrisen und beurteilte die Frühphase als positive Kontrasffolie: „Von $\times 9 \times 9$ bis 1922 beruhte die deutsche Republik auf dem freien Bündnis, das die deutsche Sozialdemokratie in den letzten Kriegsjahren mit der politischen Vertretung des katholischen Volksteils und den liberalen bürgerlichen Fraktionen eingegangen war. In diesen Jahren besaß die Republik eine einigermaßen westlichen Organisationsformen angenäherte Parlamentsregierung mit ihrem selbstverständlichen Korrelat einer politischen Machtfülle des Parlaments. ${ }^{16}$ Der Verfassungsstatus noch im Herbst $x 922$ verbuchte in der Tat auf der Haben-Seite trotz hervorgetretener Gefährdungen beachtliche Aktivposten: Nach Reintegration der verbliebenen USPDFraktion hatte die SPD nahezu wieder die Stärke der Nationalversammlung erreicht, während die KPD im Reichstag wie bei Regionalwahlen nicht viel mehr als eine Splittergruppe war. Der Rathenau-Mord hatte die republiktragende Öffentlichkeit alarmiert, und so fanden sich erforderliche verfassungsändernde Zweidrittelmehrheiten bis hin zu Stresemanns DVP: gleichermaßen für das Gesetz zum Schutz der Republik wie auch die Amtszeitverlängerung des Präsidenten Ebert ohne Volkswahl.

$1_{4}$ Carl Schmitt, Verfassungslehre, München 1928, S. 32.

is Zur Gesamtinterpretation vgl. jetzt auch Christoph Gusy, Die Weimarer Reichsverfassung, Tübingen 1997.

16 Otto Kirchheimer, Die Verfassungslehre des Preußen-Konflikts (1932), in: Funktionen des Stants und der Verfassung, Frankfurr a.M. 1972, S. 42. 
Ebenso paßte die vielzitierte Kampfansage des Zentrumskanzlers Joseph Wirth an die Adresse von deutschnationalen Haßpredigern; »dieser Feind steht rechts! « so gar nicht ins Klischeebild einer fahrlässig wehrlosen Demokratie. ${ }^{17}$

Dieses streitbare Republikschutzbündnis der linken Mitte überstrapazierte offenkundig auch Toleranzgrenzen der nationalliberalen Traditionswächter, und so war es ausgerechnet der »Kronjurist « Anschütz, der als Heidelberger Rektor den über sein offenes Wort gestürzten Kanzler Wirth mit folgendem Credo zurechtwies ${ }^{18}$ : "Der Feind steht nicht links und nicht rechts, er steht am Rhein; da steht er, der einzige, mit dem es nicht Friede noch Versöhnung gibt«; die politische Beteiligung der Volksmassen war für Anschütz wesentlich ein Mittel zur Stärkung des Nationalstaats und einer "Vaterlandsliebe", die "selbst heilig ist, so auch der $\mathrm{HaB}$, den sie fordert", den er gegen den »Erb- und Todfeind im Westen « gerichtet sehen wollte - jenes Frankreich, das er voller Abscheu nicht einmal namentlich erwähnte. ${ }^{19}$ Nur wenige Zeitgenossen erkannten, daß im Kanzlerwechsel von Wirth zu Wilhelm Cuno, dem rechtsstehend parteilosen Direktor der Hapag, der Marsch in eine andere Republik begonnen hatte; er wurde für die Weimarer Verfassungslage noch folgenreicher als jener »Marsch auf Rom «, der nur wenige Tage zuvor dem Diktaturgerede rechtsaußen erstmals Realitätsgehalt verschaffte. Über den von Anschütz als Nationalsymbol bemühten Rhein hinaus griff die französische Besatzungsmacht erst Monate später nach Konflikten um Lieferrückstände; daraufhin trieb der teilweise im "Geist von 1914 "verkündete "passive Widerstand " die Cuno-Regierung endgültig in den hyperinflationären Ruin. Der Ausnahmezustand der Währungszerrüttung geriet auch zum Einfallstor jener Notverordnungs- und Ermächtigungspraxis, die normenwidrig reguläre parlamentarische Gesetzgebungswege entmündigte. ${ }^{20}$

Auf staatspolitischer Ebene verstärkten sich parallel dazu Desintegrationsprozessc im Reich-Länder-Verhältnis. Dabei waren zum Scheitern verurteilte Lokalaktionen wie der Münchener Hitler-Putsch der NSDAP oder ein Hamburger Aufstand der Thälmann-KPD nur Randphänomene. Tatsächlich eine folgenreiche Einseitigkeit des Gebrauchs der Reichsgewalt enthielt aber ein Mißverhältnis zwischen der Reichsexekution gegen Sachsen und vorheriger Hinnahme bayerischer Übergriffe, die Preuß sogleich öffentlich anprangerte: "In Sachsen haben einzelne Mirglieder der Landesregierung zum Widerstand gegen die Reichsregierung aufgefordert; in Bayern hat die gesamte Landesregierung als solche den Maßregeln der Reichsregierung unter mehrfachem Bruch der klarsten Bestimmungen der Reichsverfassung und mit Hilfe militärischer Meuterei tatsächlich Widerstand geleistet. Indem die Reichsregierung dagegen nicht auf Grund des Artikels 48, I vorgegangen ist, hat sie seiner Anwendung

17 Vyl. dazu auch Christoph Gusy, Weimar - die wehrlose Demokratie? Verfassungsschutzrecht und Verfassungsschurz in der Weimarer Republik, Tubingen 1991.

18 Gerhard Anschuic, Drei Leirgedanken der Weimarer Reichsverfassung, Tubıngen 1923, S. 31-34 (Redetext v. 22. II. 1922).

19 Wer dies als rechtswissenschaftlich belanglose tagespolitische Außerung bagatellisieren wollte, mußte erklaren konnen, weshalb der ansonsten eher sachlich-nüchterne Kommentartext von Gerhard Anschüz, Die Verfassung des Deutschen Reichs vom 11. August 1919, x1. Aufl. Berlin 1929, die in Art. I48 WRV formulierte Richtlinie, »die es als Aufgabe unserer Schulen hezeichnet, für Völkerversöhnung zu wirken, einen Gewissenszwang gegen jeden, der sein Deutschtum hochhàlt«, zu nennen sich nicht verkneifen konnte und im Hinweis auf das "Friedensdiktat von Versailles« eine uberaus bekenntnishafte Gegenposition an die Stelle der Normauslegung setzen wollte: „Vielmehr fordert der Geist unseres Volkstums, allem andern zuvor, Erzichung der Jugend zur Deutschgesinnung, zum nationalen Selbstbewußtsein, zu nationalem Ehrgefühl« (S. 593 f.). Die Berufung auf den naturrechtlich vorgeordneten $*$ Geist unseres Volkstumsa auch gegen die positivierte Norm war sonst mehr eine Domäne der antiformalistischen *Neuerung $\approx$ der Staatsrechtlehre - doch auch Rechtspositivisten bedienen sich eben zuweilen überpositiver Argumente, wann sie mit den jeweiligen Normgehalien in Dissens geraten sind.

$20 \mathrm{Vgl}$. Achim Kurz, Demokratische Diktatur? Auslegung und Handhabung des Artikels 48 der Weimarer Verfassung 1919-1925, Berlin 1992. 
gegen Sachsen selbst die verfassungsrechtliche Voraussetzung entzogen; denn die prinzipielle Gleichberechrigung der Länder im Verhältnis zum Reich ist ein unbestrittener Rechtsgrundsatz der Reichsverfassung. " ${ }^{21}$ Bis hin zur Fluchthilfe für steckbrieflich gesuchte rechtsterroristische Mörder reichten die Gesetzlosigkeiten unter einer republikfeindlichen bayerischen Staatsregierung; das war der Ursprungsintention nach geradezu der klassische Anwendungsfall gemäß Art. 48 Abs. I WRV. Dieser verfassungskonforme Gebrauch war jedoch vom Reichswehrveto blockiert; statt dessen fand wegen einzelner kommunistischer Landesminister der Rückgriff auf kaiserzeitliche Muster des "Belagerungszustands * unter Militärbefehlshabern statt. Insofern mußte den Kritikern jeglicher Ausnahmebestimmung seitens des Verfassungsautors nunmehr explizit beigepflichtet werden: "Auf Grund des Artikel 48 und des Ermächtigungsgesetzes wird in der Strafjustiz und auf anderen Gebieten das Fundament des Rechtsstaates zertrümmert. Wenn diese Maßregeln wirklich mit der Wiederherstellung der öffentlichen Sicherheit und Ordnung und der Not des Volkes zu rechtfertigen sind, so kann man damit schließlich auch den Umsturz der Reichsverfassung im Sinne der bayerischen Forderungen auf dem Wege der Notverordnung rechtfertigen. « ${ }^{2.2}$ Das Fazit dieser eindringlich warnenden Situationsanalyse lautete: "Die Feinde der Verfassung von Weimar sehen in all diesen Wirren den Beweis für deren Fehlerhaftigkeit und Revisionsbedürftigkeit. Nun taugt die beste Verfassung nichts, wenn sie von ihren berufenen Vollstreckern falsch oder dilettantisch angewendet wird. «"s Dem ist aus heutigem Kenntnisstand nur wenig hinzuzufügen.

IV.

In einem nächsten Gedankenschritt ist die universitäre Staatsrechtslehrc auf den Prüfstand ihres verfassungsintegrierenden bzw. -desintegrativen Profils zu bringen. Der einflußreichste Kommentator Anschütz zeigte sich bereits als Exponent des nationalliberalen Kontinuitätsdenkens im Gewande des Rechtspositivismus. ${ }^{24}$ Die antipositivistische Gegenströmung ist vornehmlich an dem schulenbildenden Werk Rudolf Smends aus dem Jahre 1928 festgemacht worden. Die Rechtspositivisten waren im Urteil von Smend durch »innere Staatsfremdheit " geprägt; deren historisch-soziologische Flankierung aus den Schriften Meineckes und Webers nannte er "liberal im Sinne letzter innerer Unbeteiligung am Staat «. ${ }^{25}$ Als eine "Fundgrube " für seine Integrationslehre erschien Smend hingegen die "Literatur des Faschismus", die sich des lntegrationsproblems sin einer von unendlicher Reflexion getragenen $\mathrm{Be}$ wegung planmäßigen Aufbaus einer neuen Volks- und Staatsgemeinschaft bewußt geworden ist «. Ausgerechnet vor dieser Kontrastfolie des Mussolini-Regimes formulierte Smend wenige Zeilen darauf sein Verdikt über die Weimarer lntegrationsschwäche: "Es ist bezeichnend, daß Verfassungsgeber theoretischer Herkunft, wie

21 Hugo Preuß, Sachsen, Bayern und die Reichsverfassung, in: Ders., Um die Reichsverfassung von Weimar, Berlin 1924, S. 40 (Originaltext in: 8.Uhr-Abendblatt 30. 10. 1923).

22 Hugo Preuß, Reichsverfassung und Reichsregierung, in: Ders. (Fn. 2r), S. ins (Originaltext in: Berliner Tageblatt 24. I. 1924).

23 Preuß, Sachsen (Fn. 21 ), S. 42.

24 Wie bekanntlich auch C. Schmitx - mit seincr Lesart der intcrpretationsleitenden Antithese zum »Feind des $\times$ Bolschewismus - wollte der Standardkommentar von Anschutz, Verfassung (Fn. 19), S. 608 f., die Rechtskontinuitat zur Vorkricgsordnung im Hinblick auf Kerninstitutionen behaupten: "Wie jene anderen Grundpfeiler unserer burgerlichen Rechts-, Geseilschafus- und Wirtschaftsordnung - die Ehe, das Elternrecht, die Vertragsfreiheir, das Erbrecht - durch die Art. 1 19, 120, 152, 154, so hat durch Art. I53 $\Lambda$ bs. 1 das Privateigentum als Institution eine verfassungsmäßige Sicherung urfahren."

is Smend, Verfassung (Fn. 6), S.3. 
die von Weimar, das hier liegende erste Problem einer Verfassung übersehen haben im Kontrast dazu wurde sogleich die "Bismarcksche Verfassung « als "ein zwar unreflektiertes, aber vollkommenes Beispiel einer integrierenden Verfassung « herbeizitiert. ${ }^{26}$

Die in Wirklichkeit teilweise hochgradig desintegrative Innenpolitik der Bismarckära, vom antiliberalen Verfassungskonflikt über den antikatholischen Kulturkampf bis zum Sozialistengesetz, wollte Smend immer noch als eine "im Grundsatz sehr berechtigte "Herrschaftsintegration verteidigen, wenn er schrieb: „Mangelnde politische Wertgemeinschaft und damit zugleich mangelnden politischen Integrationswillen hat Bismarck den sstaatsverneinenden im Gegensatz zu den 'staatsbejahenden' Parteien vorwerfen wollen $\ll .{ }^{27}$ Diese angeblichen "Reichsfeinde « der Bismarckära waren aber jene Linksliberalen, Zentrumskatholiken und Sozialdemokraten, die sich inzwischen als Weimarer Verfassungsparteien formiert hatten, ohne daß Konservative wie Smend diesen Rollenwechsel akzeptierten. Nicht zufällig firmierte die Hindenburg 1925 ins Amt bringende Allianz der Republikgegner von rechts als »Reichsblock" - in historischer Anspielung auf Bismarcks »Reichsfreunde«-Kartell, um insoweit die knapp überstimmte Weimarer Koalition wieder in eine unpatriotische Ecke der Internationalisten, Kosmopoliten und Ultramontanen abzudrängen.

Die erwähnten Positionsbestimmungen sind kaum erstaunlich, wenn mitbedacht wird, daß Smend als Mitglied der verfassungsgegnerischen Deutschnationalen (bis 1930) kaum ernstlich zum Anwalt der Weimarer Neuordnung taugte und stattdessen für deren Rückbildung zur *Hindenburg-Republik « repräsentativ war. Der von Smend heftig bemängelte "Agnostizismus «, "Formalismus" und »Nihilismus des positivistisch-liberalen Verfassungsdenken ${ }^{28}$ ist außerdem nicht losgelöst von seiner These einer "Verwandtschaft zwischen der Integrationsbindung an den Staat und der religiösen an die Gottheit» zu begreifen, derzufolge "Staatsleben als Ganzes nicht eine Summe, sondern eine individuelle Einheit, eine Totalitat ist, bestimmt durch die Konkretisierung objektiver Wertgesetzlichkeiten «. Diese stets etwas kryptischen Formulierungen ließen weltanschauliche Hintergründe erkennen, wenn Smend den Standpunkt unterstützte, daß eine "Rationalisierung des politischen Denkens, die die Erfassung des politischen Gehalts als Glaubensgehalt ausschließt, damit zugleich jede politische verbindliche Gestalt in Frage stellt ${ }^{29}{ }^{29}$ Aus seinem Verdikt: "liberale Staatsform, d. h. Parlamentarismus, ist keine Staatsform «, artikulierten sich nicht allein konservative Anhänglichkeiten zum monarchischen Legitimationstypus, sondern auch glaubensprotestantische Vorbehalte gegen die weltanschauliche Neutralität der Weimarer Republik. Das Integrationsangebot der Weimarer Verfassung wies Smend ausdrücklich zurïk, wenn er aus eigener Gegnerschaft schrieb: „Die Lösung der Flaggenfrage, gewisse unitarische Überspannungen und Grundrechtsinhalte sind eher eine Belastung als eine Fördenung der Einheit und Kraft des Ganzen. ${ }^{30}$ Hinter der Annahme, daß die Weimarer Verfassung zu wenig integrieren

26 Ebd., S. ${ }_{3}$ f. - In apologetischer Schulen $\alpha$-Rezeption wird die systematısche Empfanglichkeir Smends für den faschistischen Integrationstypus gern als lediglich tllustratıver Verweis bagatellisiert. Indem Smend (Ebd., S. 70 f./Anm.) aber, im Rückgriff auf die miebendige Totalitȧt« be $\mathrm{Hegel}$ und die "Lebendigkeit des sitclich aufgefaßten Staats « bei Stabl, die eigene Singularitat betont: wIn der deutschen Staarstheoric seit Mitte des 19. Jahrhunderts steht die hier vertretene Anschauung, soweit ich schc, allein", ist die einzigc parallelisierende Bezugnahme höchst aufschlußreich: „Eine entschiedene Wendung zu einer antistatischen Theoric von Nation und Staat hat M. H. Boehm angekündigt (Rundbriefe 4 /s des Instituts für Gren\%- und Auslandsdeutschtum, Okt./Nov. 1926) $;$; jener M. H. Bochm gehort nun aber ganz eindeutig zur intel-

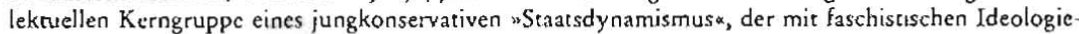
komponenten durchsetzt war.

27 Ebd., S. 40 (Anm).

28 Ebd., S. 3, S, 13.

29 Ebd., S. 50 (Anm.), 47, so 
konnte, verbarg sich politisch also nicht selten die Tatsache, daß sich frühere Nationalliberale nur partiell und Konservarive eher gar nicht in die so definierte neue Ordnung integrieren wollten. ${ }^{3 t}$ In systematischer Hinsicht vermochte Smend wenig zum Thema "Integration durch Verfassung " beizutragen; vielmehr sieht er umgekehrt Verfassung, und Staatlichkeit überhaup ${ }^{32}$, auf homogenisierend vorgeordnete ${ }^{33}$ Herrschafts- und Glaubensintegration angewiesen. Auch in Carl Schmitts dezisionsgeordneter Souveränitätslehre ${ }^{34}$ und sogar Hermann Hellers etatistisch konzentrierter "Einheit der Gebietsentscheidung « ist übrigens der Normenintegration, jeweils in polemischer Distanz zu Kelsen, nur Sekundärbedeutung zugedacht. ${ }^{35}$

V.

Das rechts- und politiktheoretisch bemerkenswerteste Stichwort der Smendschen Integrationslehre, die "fließende Geltungsfortbildung des gesetzten Verfassungsrechts ${ }^{36}$, mochte unter dem halbautoritären System des Kaiserreiches teilweise noch ein Reformfaktor im epochalen Institutionenwandel gewesen sein: Es hatten sich beispielsweise gegen landesfürstliche Intentionen aus dem Nukleus der Reichsämter schrittweise die Konturen einer Reichsregierung als notwendiges Gegenüber des Reichstags abgezeichnet. Eine parlamentarische Demokratie bedurfte nur ein Jahrzehnt nach ihrer Konstituierung hingegen zuallererst der Geltungsbekräftigung ihrer Verfassungsnormen. Gleichwohl stimmten Ende der zoer Jahre große Teile der Staatsrechtslehrer in der Grundtendenz mit dem Leitsatz "Mehr Macht dem Reichspräsidenten» überein, auch wenn dies als deutschnationale Wahlparole instrumentalisiert war. Ein selten thematisierter Hintergrund dafür, weshalb die Staatsrechtslehrerzunft entgegen dem Verfassungswortlaut das Reichskanzleramt geradewegs degradierte, war parteipolitischen Zuschnitts: Mir Ausnahme dreier Monate im Herbst 1923, als Stresemann dic Konkursabwicklung des Ruhrkampfes und der Hyperinflation anvertraut war, hatten sämtliche Weimarer Kanzler mit Parteizugehörigkeit eine »rote o oder »schwarze «infärbung: Sozialdemokraten und Zentrumskatholiken waren jedoch in der Staatsrechtswissenschaft noch weniger einflußreich als DDP-Liberale. Selbst das repräsentative "Handbuch des deutschen Staatsrechts",

30 Ebd., S. I12, 126.

31 In der gegen Snend gerichteten Streitschrift war insofern fur Hans Kelsen, Der Staat als Integration, Wien 1930, "der Kampf gegen den Parlamentarismus in Wahrheit ein Kampf gegen die Demokratue, mithin ein geisriger "Kampf gegen die Verfassung der deutschen Republik $(S .82,92)$.

32 Es ist fur das Eingestandnis zur eigenen Studie, daß nihre sachliche und formelle Ausgeglichenheit bceinträthtigt erscheinen muß, durchaus charakteristisch, wenn eingangs klargestellt bleiben soll * Das hier entwickelte Sinnprinzip der Integration, des einigenden Zusammenschlusses, ist nicht das des Staates überhaupt, sondern das seiner Verfassung \&, in einer grundlegenden Kapiteluberschrift hingegen "Integration als grwndlegender Lebensvorgang des Staatsa in solcber entdifferenziercnden Pauschalitat eingeführt wird; Smend, Verfassung (Fn. 6), S. VIII, 18.

33 Unter Berufung auf C.Schmitt teilte Smend, Verfassung, S. 114 , dessen Grundthese, »daß Demokratie Homogenitat voraussetzt $\alpha$ - in derlci substantialistischen Dogmen ihrer politischen Theologien und niche der »geisteswissenschaftlichen« Alternative zum Positivismus liegt die zentrale Differenz zu Kelsen und Preuß, die eine moderne Verfassungsdemokratie gerade als prozedurales Integrationsdach uber ciner sozioökonomisch und soziokulturell heterogenen Gescllscbaft, d. h. auf Pluralitaten bzw. Antagonismen begrundeten.

34 Diesbezüglich einschlagige Passagen finden sich bei Carl Schmin, Soziologie des Souveränitätsbegriffs und politische Theologie, in: Hauptprobleme der Soziologie. Erimerungsgabe für Max Weber, Bd. 2, Munchen 1923 , S. $3-35$.

35 Dazu grundlegend und mit - im Unterschied zur posthumen "Staatslehre $\alpha$ (Leiden 1934) - noch mehr Gemeinsamkeiten zu den Arupositivismen von Schmitt bis Smend vor allem: Hermann Heller, Die Souveranitat. Ein Beitrag zur Theorie des Staats- und Volkerrechts, Berlin 1927.

36 Sinend, Verfassungstecilit, S. I38. 
herausgegeben von Anschütz und dem teilweise dezidierter liberaldemokratischen

Richard Thoma, blieb nicht von einer verfassungsfremden Lehrmeinung verschont, zwischen Präsident und Kanzler »das Vertrauensverhältnis des Oberführers zum selbständigen Führer zu konstruieren. ${ }^{37}$ Der Weltkriegsoffizier Heinrich Brüning mag 1930 seine Dienststellung als Reichskanzler gegenüber dem Generalfeldmarschall a.D. Hindenburg derart nachgeordnet empfunden haben; mit solchem Hineinragen militärischen Hierarchiedenkens ${ }^{88}$ in die Zivilrepublik begannen jedoch die fließenden Übergänge von der Weimarer Demokratie zu einem allenfalls noch halbparlamentarischen Präsidialregime.

Vollends gar nichts mehr gemein mit der Verfassung von I9 Ig hatten jene Entwicklungen des Jahres 1932, die allzu häufig zum Beleg für desintegrierte »Weimarer Verhältnisse" angeführt worden sind. In diesem letzten Jahr vor dem Übergang zum NS-Regime standen lediglich ein Dutzend Reichstagssitzungen nicht weniger als fünf Dutzend Notverordnungen gegenüber. Dieses Mißverhältnis übertraf bei weitem die außen- wie innenpolitische Krisenlage des Jahres I 923 und entsprach der endgültigen Verabschiedung von parlamentarischer Demokratie. Die Wiederwahl Hindenburgs im April 1932 mit den Stimmen der großen Mehrzahl seiner Gegner von 1925, einzig in Sorge vor sofortiger Machtübernahme Hitlers, bedeutete im Ergebnis den politischen Selbstmord der Verfassungsparteien aus Furcht vor dem NS-Todesurteil: Die anschließende Einsetzung des Papen-Regimes mit Auflösung des Reichstags aus dem offen bekundeten Motiv, dic Volksvertretung an normenverbürgter Aufhebung von Notverordnungen zu hindern, war glatter Verfassungsbruch, ebenso wie daraufhin inszenierte Staatsstreichaktionen gegen das preußische Kabinett der Weimarer Koalition. Eine derartige antiparlamentarische Kampfregierung wie Papens »Kabinett der Barone " hatte es außerhalb von Kriegsjahren zuletzt vor sieben Jahrzehnten im preußischen Verfassungskonflikt gegeben. Was insofern mit dem Rückschritt in staatsautoritär bedrohte frühkonstitutionelle Verhältnisse begann, endete mangels eigener Massenintegrationsbasis diescr vorindustriellen Eliten mit ciner fataler Entwicklungslogik in der postkonstitutionellen NS-Diktatur. ${ }^{39}$

$V I$.

Mit zählebigen Klischeebildern einer desintegrativen Weimarer Verfassung konnte aber die Mehrzahl jener Staatsrechtslehrer, die vor 1933 nicht als Verfechter der parlamentarischen Demokratie galten, ihre nach 1945 fragwürdig erscheinende Grundhaltung bequemer rechtertigen. Noch aus der Rückblende hat ein prominenter Fachvertreter die fortwirkende "Abneigung gegen ein uns innerlich fremdes

37 Fritz Poetzsch-Heffter, Organisation und Geschätsformen der Reichsregierung, in: Gerhard Anschutz/ Richard Thoma (Hrsg.), Handbuch des Deutschen Staatsrechts, Bd. I, Tubingen 1930, S. 514; bei Anschutz, Verfassung (Fn. I9), S. 282, bewahrt sicb der Rechispositivismus immerhin zugunsten einer normengercchten Berucksichtigung von Art. 56 WRV: ${ }^{2}$ Damit ist der Reichskanzler über die andern Minister bedeutsam hinausgehoben und ihm dic Stellung eines nicht für die Linzelheiten, sondern fur das Ganze verantwortlichen leitenden Statsmannes angewiesen* - dem gegenuber es selbstverstandlichen keinen "Oberführer* geben kann.

38 Der prasidiale "Oberbefehl uber die gesamte Wehrmacht des Reichs« (Art. 47 WRV) begrundete keinen politischen *Oberführer $\alpha$-Status, da Art. so lautete: "Alle Anordnungen und Verfügungen des Reichsprasidenten, auch solche auf dem Gebiete der Wehrmacht, bedurfen zu ihrer Gultigkeir der Gegenzeichnung durch den Reichskanzler oder den zuständigen Reichsminister «; nur ein auf seine Richtlinienkompetenz gemaß Art. s6 verzichtender Kanzler war zum bloßen "Abzeichner subordinierbar.

39 Eine Gesamtinterpretation zur historisch-politischen Entwicklung wird demnächst vorliegen: Declef Lehnert, Die Weimarer Republik. Parteienstaat und Massengesellschaft, Suttgart 1999. 
abstraktes Schema der Demokratie« bestätigt, und er nannte gar »das Bedürfnis der Anhänger des Dritten Reichs nicht ganz ungerechtfertigt, zu einer gerade für Deutschland und in dieser Lage gültigen ganz konkreten politischen Ordnung und Ausrichtung zu kommen «. Dieser Staatsrechtler hieß nicht etwa Schmitt, wie die Tendenz dieser Formulierungen vermuten lassen könnte, sondern wohlbemerkt Smend ${ }^{40}$; er ist insofern für Beurteilungen in und zu der Weimarer Periode auch nicht anders einzustufen. Bemerkenswert ist hingegen die abweichende Haltung von Erich Kaufmann: Zwar hat er als traditioneller Konservativer, in später Nachfolge Stahls und bezugnehmend auf die Institutionenlehre Haurious, zuerst einen werteblinden Normenformalismus der Rechtspositivisten attackierr ${ }^{4}$; doch vollzog er schon Mitte der zoer Jahre den Bruch mit den Deutschnationalen und verteidigte zunehmend die Weimarer Legalität. Insofern weniger von früherer Mitverantwortung an delegitimierenden Lehrmeinungen belaster, äußerte sich ein als liberal-konservativ einzustufender Kaufmann nach 1945 im gleichen Sinne wie linksliberale oder sozialdemokratische Außenseiter der eigenen Disziplin: "Die Weimarer Verfassung als solche war gar nicht so schlecht, wie man heute vielfach behauptet. Das deutsche Volk hat unter dieser Verfassung von r 919 bis, sagen wir, $1930 \mathrm{im}$ wesentlichen gut leben können. Aber die politischen Faktoren haben die Situation nach 1930 nicht mehr zu meistern vermocht. " ${ }^{2}$ Diesem Fazit läßt sich - mit zusätzlichem Hinweis auf negative Präzedenzfälle in der Inflationskrise um das Jahr 1923 - durchaus beipflichten.

Seither konnte sich bundesdeutscher Verfassungspatriotismus, allerdings erst über den Weimar verwehrten Bewährungszeitraum eines Vierteljahrhunderts, nicht allein kompensierend zur geteilten Nation herausbilden, sondern auch im Hinblick auf den Symbolwert des Grundgesetzes für demokratische Stabilität und gesellschaftliche Prosperität. Justizminister Radbruch hatte schon zu den ersten größeren Verfassungsfeiern im August 1922 den Versuch unternommen, die Weimarer Verfassung als »unsichtbares Vaterland « zu propagieren und ein neugeordnetes "schwarzrotgoldenes Vaterland " gegen den kaiserzeitlichen Nationalismus abzusetzen. ${ }^{43}$ Dieses Integrationsangebot scheiterte faktisch nicht an damals noch wenigen lärmenden Völkischen zur Rechten und Kommunisten zur Linken, sondern an den Erben der Konservativen und Nationalliberalen; deren Wortführer und Anhänger begingen weiterhin "ihren « schwarzweißroten Reichsgründungstag und verweigerten sich, "Deutschland über alles" singend, den alljährlichen Verfassungsfeiern.44 Aus einem Integrationsverständnis, das einseitig konsensorientiert und zu wenig konfliktpluralistisch ausgelegt ist, wurde das Problem des politischen Extremismus für die Zerstörung von Weimar zumeist überschätzt; dieses Gewaltpotential wäre sogar in den Krisenjahren sicherheitspolizeilich beherrschbar geblieben, hätte sich nicht innerbalb der Träger und Garanten des staatlichen Gewaltmonopols selbst eine verfassungsferne Haltung breit gemacht: allen voran unter Reichswehroffizieren, jedoch auch in

40 Rudolf Smend, Das Problem der Presse in der heutigen geistigen Lage (1946), in: Ders., Staatsrechtliche Abhandlungen und andere Aufsatze, Berlin r $955, \mathrm{~S} .386$.

4 I Da Kaufmann trotz seincs (auch von Smend anerkannten) überlegenen Bildungshorizonts eine traditionspflegende "Schule* versagt geblieben ist, zumal er in der zweiten Halfte der $20 e r$ Jahre als Rechtsberater der Außenpolitik Stresemanns außeruniversitär starker engagiert war, kann abgesehen von den "Gesammelten Schriften“ Kaufmanns (3 Bde./Göttingen 1960) nur auf eine vergleichende Studic verwiesen werden: Klaus Rennert, Die sgeisteswissenschaftliche Richtung* in der Staatsrechtslehre der Weimarer Republik. Untersuchungen zu Erich Kaufmann, Günther Holstein und Rudolf Smend, Berlin 1987.

42 Erich Kaufmann, Grundtatsachen und Grundbegriffe der Demokratie, München 195 I, S. I 7.

43 Vorwärts, 12.8.1922.

$44 \mathrm{Vgl}$. dazu Detlef Lehnert/Klaus Megerle (Hrsg.), Politische Identitat und nationale Gedenktnge. Zur politischen Kultur in der Weimarer Republik, Opladen r989. 
der hohen Bürokratie und bei vielen Richtern. Die besatzungsrechtliche Zerschlagung des NS-Herrschaftssystems konnte in dieser Hinsicht solche Restaurationskräfte gründlicher schwächen als der Regimewechsel in der Novemberrevolution rgr 8.

Ein für die Weimarer Epoche am stärksten kompatibles Nachbarland illustriert eindringlich das Bezugsverhältnis zwischen dem Integrationsstatus eines massengesellschaftlichen Parteiensystems und jenem der Verfassungsordnung. In Österreich gelten bis heute die Verfassungsnormen aus der Ersten Republik; so konnte man dort nicht auf die eigentümliche Idee kommen, einem textidentischen Verfassungswerk einerseits die politische Lager-Konfrontation der späten $20 e r$ und frühen zoer Jahre und andererseits die Konkordanzdemokratie nach r945 zuzuschreiben. ${ }^{45}$ Die 1929 sogar noch in Richtung Weimar revidierte Kelsen-Verfassung wurde in Österreich nach 1945 nicht von desintegrierter, vielleicht cher schon von überintegrierter Staatspraxis mit Großen Koalitionen und sozialpartnerschaftlichem Korporatismus begleitet. Wenn es demnach entscheidend auf die Geltungsbedingungen der parteiendemokratischen Realverfassung ankommt, spricht wenig gegen die Annahme, daß eine reaktivierte Weimarer Verfassung seit 1949 in der Bundesrepublik Deutschland instrumentell weithin problemlos "funktioniert« hätte; sie durfte aber wegen ihres symbolischen Erinnerungsgehalts des historisch-politischen Scheiterns an Gegenkräften lediglich in einer umfassend redigierten Neuauflage namens "Grundgesetz» zurückkehren. Dessen bevorstehender so. Jahrestag sollte endlich Gelegenheiten eröffnen, veraltende Fragestellungen zur Weimarer Republik mit neuakzentuierten Antworten zu verschen - und dabei Verknüpfungspunkte beider demokratischer Verfassungen nicht länger wie ein Staatsgeheimnis zu hüten.

Monika Brakhage/Regine Drewniak

\section{,Sonst wäre ich im Knast gelandet ....6}

Die ambulanten Maßnahmen aus der Perspektive der betroffenen Jugendlichen

Ambulante sozialpädagogische Maßnahmen werden als spezialisierte Jugendhilfeangebote für junge Straffällige bereitgestellt, um traditionelle jugendgerichtliche, vor allem freiheitsentziehende Sanktionen durch pädagogische Unterstiltzungsleistungen zu ersetzen.

Trot jahtr/chntelanger Firfahrung nit entsprechenden Projekten mangelt es bislang an konkreten Inlümationen über die Ausgestaltung der Angebote selbst sowie deren Beurteilung durch die erreichte Zielgruppe. Im Mittelpunkı dieser Untersuchung stehen die Perspektiven von 48 intcrviewten Jugendlichen und Heranwachsenden aus nicdersächsischen Projekten zu ihren Teilnahmeerfahrungen und Teilnahmebeurteilungen.

Über ihren Beitrag zur erforderlichen größeren Transparenz. dieser Angeborsformen hinaus bieten die Befunde eine Grundlage für die notwendigerweise fortzuführende Debatte über eine zielentsprechende Praxis in Jugendhilfe und Jugendgerichtsbarkeit in Umgang mit jungen Straffälligen.

1999. I63 S., brosch., 32,-DM, 234,- öS, 29,50 sFr, ISBN 3-7890-6063-1 (Interdisziplinäre Beiträge zur Kriminologischen Forschung, Bd. 15)

\section{$\checkmark$ NOMOS Verlagsgesellschaft 76520 Baden-Baden}

4) Vgl. den Überblick bei Anron Pelinka/Manfried Welan, Demokratic und Verfassung in Österreich, Wien 1971. 